\title{
IgE in asthma and atopy: cellular and molecular connections
}

\author{
Hans C. Oettgen and Raif S. Geha \\ Division of Immunology, Children's Hospital, Harvard Medical School, Boston, Massachusetts 02115, USA
}

Address correspondence to: Hans C. Oettgen, Division of Immunology, Children's Hospital, 300 Longwood Avenue, Boston, Massachusetts 02115, USA. Phone: (617) 355-7768; Fax: (617) 355-8205; E-mail: oettgen@a1.tch.harvard.edu.

The atopic triad. Allergists are frequently faced with patients simultaneously suffering from 3 diseases asthma, atopic dermatitis, and allergic rhinitis, a constellation recognized as the "atopic triad." The clinical association of these conditions, as well as their cosegregation in affected family members within allergic pedigrees, has long suggested common underlying pathophysiologic mechanisms. The term atopy was coined in the 1920 s by Arthur Fernández Coca, founder of The Journal of Immunology, and Robert Anderson Cooke, who introduced intracutaneous skin testing. Atopy, meaning "strange disease," was used by Coca to describe antigenspecific reactions with apparent immunological specificity for which no precipitating antibodies could be identified in plasma. The instigating antigens were called atopens, and the mysterious plasma factor that conferred hypersensitivity was labeled atopic reagin.

Only much later in this century was IgE identified as the reagin. $\mathrm{IgE}$ is set apart from the other immunoglobulin isotypes by its very low plasma levels and short halflife. In addition to triggering immediate-hypersensitivity reactions and late-phase responses, there is accumulating evidence that preformed IgE can augment humoral and cellular immune responses to allergens. IgE blockers have recently been developed and show some promise in the treatment of allergic diseases. The production of $\operatorname{IgE}$ is tightly regulated and involves a complex network of cellular and molecular signals. As the millennium draws to a close, it is clear that IgE production represents only one feature of a larger specific immune response orchestrated by the Th 2 subset of CD $4^{+}$Th cells. Th 2 cells are critical in maintaining both the state of chronic and relapsing eosinophil-predominant inflammation and the acute hypersensitivity responses characteristic of the atopic diseases. Here we will give a brief overview of the roles of $\operatorname{IgE}$ in allergic pathophysiology, and the molecular and cellular factors that ultimately regulate IgE production and Th2 expansion.

IgE functions in allergic responses: immediate bypersensitivity, allergen focusing, and IgE receptor regulation. Asthma, allergic rhinitis, and atopic dermatitis are almost invariably accompanied by elevated levels of IgE. Genetic analyses of families have shown that bronchial hyperresponsiveness (BHR) and IgE levels are linked (1). Thus, in clinical practice, allergen-specific IgE (as demonstrated by skin testing or in vitro assays) is generally believed to be inextricably connected to the induction of allergic airway symptoms, and is used as a guide for environmental modification and immunotherapy. The interaction of $\operatorname{IgE}$ with antigen is known to lead to a variety of immunological sequelae. Cross-linking of IgE bound to mast cells by FceRI triggers the release of preformed vasoactive mediators, synthesis of prostaglandins and leukotrienes, and the transcription of cytokines. In the bronchial mucosa, these mediators of immediate-hypersensitivity reactions rapidly induce mucosal edema, mucous production, and smooth muscle constriction, and eventually elicit an inflammatory infiltrate.

IgE-induced mast cell degranulation in vivo is often followed by a late-phase reaction (LPR), a second wave of hypersensitivity responses occurring many hours after the acute reaction and dependent upon eosinophils. In asthmatics, this manifests as a second wave of decreased airflow 4-8 hours after the initial allergen contact. IgE antibodies (but not other isotypes) are capable of passive transfer of both acute and LPR sensitivity to allergen challenge. It has been postulated that chronic airway symptoms result from persistent late-phase inflammatory responses in situations of perennial allergen exposure. Evidence in humans and animal models suggests that IgE-mediated mast cell activation gives rise to both the acute and late-phase responses. Acute responses are accompanied by evidence of mast cell activation and mediator release. Inhibition of mast cell activation, or interference with the function of the mediators, blocks the onset of both phases of the response.

In addition to activating mast cells via FceRI, IgE interacts with other receptors, including CD23 (FcERII). CD23$\mathrm{IgE}$ interaction provides an important mechanism whereby allergen-specific IgE can augment cellular and humoral immune responses in settings of recurrent allergen encounters (Figure 1). Passive sensitization of murine or human B cells with IgE greatly enhances their ability to present antigen and induce responses by specific $\mathrm{T}$ cells. This effect is CD23-dependent. The in vitro production of IgE and IgG antibodies is also amplified in a CD23dependent manner by the presence of antigen-specific IgE.

Augmentation of immune responses by IgE, initially observed in vitro, has been documented in vivo as well. Mice injected intravenously with antigen mount a significantly greater IgG response when the original immunogen is accompanied by an intravenous dose of antigen-specific $\operatorname{IgE}(2)$. The titers of other isotypes (including IgE) are also augmented, and this effect can be completely abolished by in vivo pretreatment with anti-CD23. As expected, $C D 23^{-1}$ mice cannot display augmentation of immune responses by IgE. Such observations would predict that preformed allergen-specific IgE present in bronchial-associated lymphoid tissue might enhance active immune responses to subsequent allergen inhalation. 


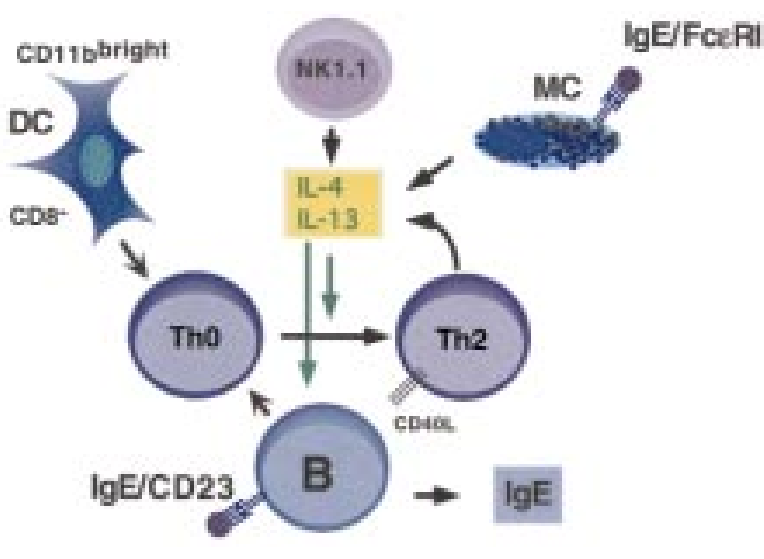

\section{Figure 1}

Cellular and cytokine feedback loops promoting IgE production in the allergic respiratory mucosa. APCs, preformed IgE, and a permissive cytokine milieu may all help predispose to Th2 responses in the bronchial mucosa of atopic individuals. Dendritic cells (DC) of the myeloid phenotype (CD8-, CD11 bright) favor the generation of Th2 cytokines. Allergen-specific IgE, usually present in atopic individuals, can passively sensitize $B$ cells via the low-affinity IgE receptor CD23 and enhance the antigen-presenting capacity of these cells by several logs. Antigen presentation by B cells favors Th2 responses. The local production of IL-4 in the bronchial mucosa by multiple cell types enhances Th2 expansion. In addition to the preestablished allergen-specific Th2 T cells resident in the bronchial mucosa of allergic patients, possible sources of IL-4 include NK1.1 cells (both $T$ and non-T) and mast cells. IL-4 production by mast cells is favored if they are sensitized, via FcERI, by preformed IgE specific for the allergen. IL-4 supports the differentiation and expansion of allergen-specific Th 2 cells, which then provide 2 signals, IL-4 and CD40L, that drive IgE production by $\mathrm{B}$ cells.

In addition to initiating immediate-hypersensitivity reactions and facilitating antigen focusing, IgE has recently been shown to have the ability to modulate the expression of its own receptors, FcERI and CD23. FcERI numbers are 4- to 5-fold greater on peritoneal mast cells and bone marrow basophils from wild-type animals than on those from $I g E^{-/-}$mice (3). Similarly, wild-type mice express approximately 4- to 6-fold more CD23 on their B cells than do $I g E^{-/-}$animals (4). Both receptors can be upregulated in vivo in the $\operatorname{Ig} E^{-/-}$mice by intravenous administration of $\operatorname{IgE}$. There is a direct correlation between FceRI density and excitability of mast cells. IgE-mediated upregulation of FcERI significantly enhances the ability of mast cells sensitized with IgE to degranulate in response to allergen challenge. Thus, IgE effects a positive-feedback mechanism that enhances immediate-hypersensitivity responses. Upregulation of CD23 by IgE could enhance allergic responses in the bronchial mucosa by enhancing antigen uptake and presentation.

Allergic pathogenesis in $\mathrm{IgE}^{-/-}$mice. Despite these putative functions of IgE in allergy, it has been difficult to demonstrate a precise role for this isotype in asthma pathogenesis using murine models of the disease. Inflammation of the bronchial mucosa and the induction of BHR are elicited to the same extent in wild-type and $I g E^{-/-}$mice subjected to repeated inhalation of allergen extracts of the mold Aspergillus fumigatus (5). Allergic rhinitis and atopic dermatitis also arise independently of the presence of IgE in mice, a surprising finding given the strong clinical correlations in humans between IgE levels and these diseases. Even the syndrome of active anaphylaxis, with mast cell activation and mediator release, can be displayed by both OVA-sensitized IgE ${ }^{-/-}$and FcERI-deficient mice after intravenous challenge with $\operatorname{OVA}(6,7)$.

Although these findings point to the existence of parallel pathways of allergic reactions, they certainly do not exclude an important role for IgE in allergic diseases in humans. The strong expression of hypersensitivity reactions in the absence of IgE may be species-specific; in mice, the IgG1 isotype effectively sensitizes mast cells and can passively confer hypersensitivity upon naive recipients. Furthermore, in the case of asthma, the animal analyses performed to date have focused primarily on aspects of the disease that may be primarily $\mathrm{T}$ cell-driven, including eosinophil recruitment and BHR. It is possible that IgE plays a greater role in acute responses to inhaled allergen, including allergen-induced bronchospasm and late-phase responses of the airways.

Anti-asthma effects of IgE blockers. The concept that allergenspecific IgE initiates acute allergic airway symptoms and promotes ongoing allergic responses has driven the development of therapeutics such as blockers of the interaction of IgE with its high-affinity receptor FceRI. These have included FceRI $\alpha$-IgG fusion proteins, as well as murine (MaE11) and humanized (E25) mAb's $(8,9)$. Humanized antibodies have been developed to avoid the potential immunogenicity of a chronically administered murine reagent. These blockers share an important property they inhibit IgE binding to mast cells, but do not cross-link cell-bound IgE or provoke mast cell degranulation.

Initial clinical studies using E25 have demonstrated a significant drop in free serum IgE levels in E25-treated patients. Total IgE concentrations, however, are elevated, presumably because of impaired clearance of IgE when complexed to E25. In a finding consistent with the observations made in $I g E^{-/-}$mice, E25 treatment markedly and reversibly downregulates basophil expression of FceRI. In bronchoprovocation challenges, E25-treated subjects have markedly decreased airflow obstruction induced by allergen inhalation. Both the early- and latephase bronchoconstrictive responses are affected with significant differences in $\mathrm{FEV}_{1}$, extending to 7 hours after allergen challenge. These findings support the notion that $\operatorname{IgE}$ is a key instigator of immediate responses to inhaled allergen.

It is not yet clear whether E25 treatment has any impact on the chronic allergic inflammation of asthma. In these initial trials, which involved relatively mild asthmatics and a short treatment period, it did not affect bronchial eosinophil recruitment, as measured in induced sputum. The effect of E25 on BHR induced by methacholine (distinct from bronchoconstriction triggered by allergen) is modest. Clinical measures of chronic asthma are not significantly different between E25-treated individuals and controls. There are no differences in peak flow scores, medication usage, or symptom scores. These data support the concept that while IgE has a critical role in acute responses to inhaled allergen, including immediate and late-phase bronchoconstriction, other allergen-driven mechanisms underlie chronic eosinophilic inflammation and BHR. The 
efficacy of E25 is now under investigation in other allergic disorders, including allergic rhinitis. In the future, it will certainly make sense to assess the effectiveness of E25 in settings of IgE-mediated immediate-hypersensitivity reactions, such as severe food-induced anaphylaxis.

Regulation of IgE production: 2 signals are required for the isotype switch. The strong association of $\operatorname{IgE}$ with the atopic diseases, and its documented role in immediatehypersensitivity reactions in the airway, has driven efforts to define the cellular and molecular interactions that regulate its production. The immunoglobulin heavy chain gene locus $(\operatorname{IgH})$, present in the genome of all naive $\operatorname{IgM}^{+} \mathrm{B}$ cells, contains all the genetic information required to synthesize each of the immunoglobulin isotypes. A cassette of rearranged V, D, and J segments located at the $5^{\prime}$ end of the IgH locus encodes the antigen-combining regions of a mature immunoglobulin heavy chain. This is followed by widely spaced clusters of exons encoding the constant-region domains of each of the heavy chain isotypes. To produce an isotype other than IgM or IgD, an active reorganization of the B-cell genome must occur in which the $\mathrm{V}_{\mathrm{H}}(\mathrm{D}) \mathrm{J}_{\mathrm{H}}$ cassette is moved into a location adjacent to the exons encoding constant domains of immunoglobulin heavy chains (isotype switch), creating a complete template for the transcription of heavy chain mRNA.

In the case of $\mathrm{IgE}$, it is now clear that 2 distinct signals, both of which can be provided by Th cells, are required to drive this process of isotype switching (Figure 2). The first signal can be provided by the cytokines IL-4 or IL13 , and stimulates transcription at the $\mathrm{C} \varepsilon$ gene locus, which contains the exons encoding the constant-region domains of the IgE $\varepsilon$-heavy chain. A second signal, delivered by the interaction of CD40L on the surface of T cells with CD40 on the B-cell membrane, activates the genetic rearrangement (called deletional switch recombination) that brings into proximity all of the elements of a functional $\varepsilon$-heavy chain.

IL-4, IL-13, their receptors, and signal transduction. IL-4 and IL-13 are the only cytokines that can support IgE production by cultured B cells. IL- 4 is produced primarily by Th2 cells, but is also derived from NK1.1+ cells, mast cells, and basophils. IL-13 is produced by activated Th2 cells, as well as by Th0 cells, mast cells, and dendritic cells. The receptor for each cytokine is multimeric. IL-4 and IL-13 receptors share the IL-4R $\alpha$ chain. The highaffinity IL-13 receptor contains the IL-4R $\alpha$ chain and an IL-13 binding chain (IL-13R $\alpha_{1}$ or IL-13R $\alpha_{2}$ ), whereas the IL-4 receptor is composed of IL-4R $\alpha$ and the common cytokine receptor $\gamma$ chain $\gamma$ c. Although the 2 cytokines exert many of the same influences on target cells, they differ in several important respects. Because $\mathrm{T}$ cells do not express IL-13 receptors, exogenous IL-13 does not promote Th2 responses or suppress Th1 differentiation in antigen-stimulated cultures in the same way as IL-4. Surprisingly, however, mice with a targeted deletion of IL-13 do have impaired Th2 responses, along with decreased IgE production and CD23 expression. This in vivo finding, suggestive of a role for IL-13 in Th2 differentiation, is supported by the observation that IL-4R $\alpha$ knockout mice (which would have defective signaling for both IL-4 and IL-13), as well as IL-4-/- IL-13-/- double mutants, have a more severe Th2 impairment than do IL-4 mutants (10). The discrepancy between tissue culture and in vivo observations suggests that IL-13 may impact Th differentiation indirectly, perhaps by modulating the function of antigen-presenting cells. The exogenous administration of recombinant IL-13 alone induces bronchial inflammation and BHR, and studies using murine asthma models and neutralizing anti-IL$13 \mathrm{mAb}$ 's or sIL-13R $\alpha_{2}-\mathrm{Fc}$ fusion protein show a marked attenuation of allergic inflammation $(11,12)$.

The IL-4R $\alpha$ and $\gamma_{c}$ chains are associated with the Janus-family tyrosine kinases JAK1 and JAK3, respectively. After ligand binding, these kinases phosphorylate tyrosine residues in the intracellular domains of the receptor chains, providing docking sites for the signal transducer and activator of transcription (STAT) molecule STAT6. These STAT6 molecules become phosphorylated and then form homodimers that translocate to the nucleus, where they bind to specific sequences (TTCN(N)GAA) in the promoters of IL-4-responsive genes, including I $\varepsilon$. The importance of STAT6 in IL-4-induced isotype switching is confirmed by the lack of $\operatorname{IgE}$ class switching in mice deficient for STAT6 (13). Because IL-4R signal transduction is also important for the induction and expansion of Th2 cells, STAT6-deficient mice have absent Th2 responses, as do IL-4 knockouts.

Like IL-4, IL-13 activates STAT6 and induces Cegermline transcripts. It does not, however, activate JAK3. As a result, IL-13R signaling is intact in B cells from JAK3-deficient patients. There is evidence that the IL13R $\alpha$ chain associates with Janus-family kinase members JAK2 and TYK2. Upon ligand binding, heterodimerization of the IL-13R chains initiates a cascade of events identical to that which follows heterodimerization of the IL-4R chains by IL-4, leading to STAT6 dimerization and nuclear translocation.

The adapter protein insulin receptor substrate-1 (IRS1) and the related protein, IRS-2, are also phosphorylated after IL-4R engagement. The intracellular domain of the IL-4R $\alpha$ contains an I4R motif that is also present in the insulin and IGF-1 receptors. Phosphorylated IRS-1 or IRS- 2 can bind to the I4R motif and then serve as a docking protein for many src-homology 2 (SH2) domain-containing molecules, thereby providing a potential link between the IL-4R pathway and other pathways involved in cell growth, including the phosphatidylinositol 3-kinase (PI 3-kinase) and mitogen-activated protein kinase (MAPK) pathways. IL-4 treatment of human B cells results in the dephosphorylation of tyrosine residues in the p85 subunit of PI 3-kinase, likely by the SH2-containing tyrosine phosphatase-1 (SHP1), which associates with IL-4R and PI 3-kinase after IL4 stimulation. The importance of SHP-1 in IL-4R signaling and IgE production in humans was recently demonstrated by the identification of a gain-of-function mutation, R576, in the intracellular domain of IL-4R $\alpha$ of atopic individuals. The R576 mutation was shown to lead to a decrease in SHP-1 binding to IL-4R $\alpha$ with a resultant increase in signaling via IL-4R (14).

Activation of the $\mathrm{I} \varepsilon$ promoter requires a number of transcription factors in addition to STAT6. The promoter contains binding sites for nuclear factor- $\mathrm{KB}$ (NF- 


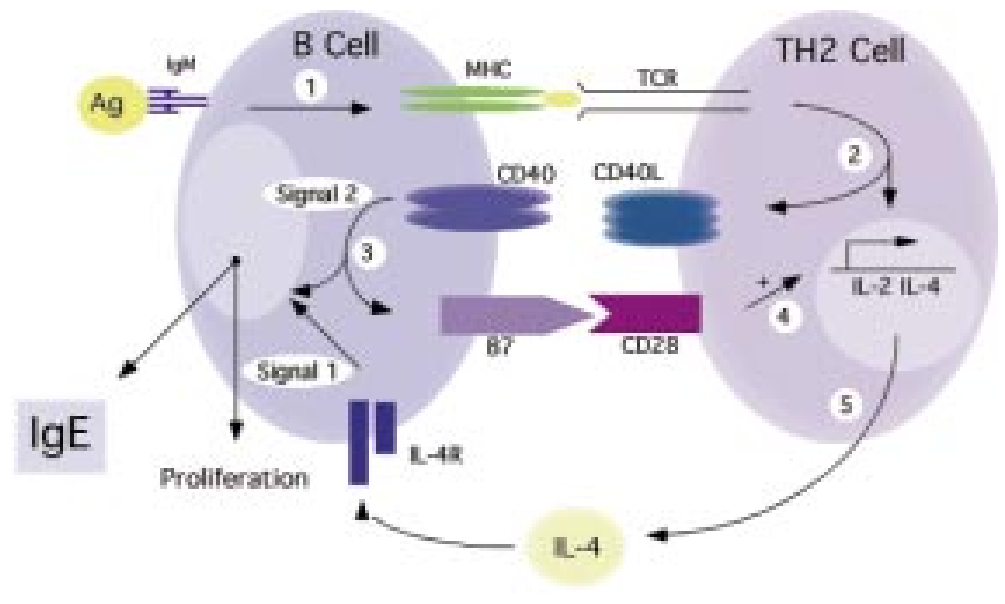

Figure 2

T- and B-cell interaction: 2 signals for IgE production. IgM-facilitated uptake of allergen ( $\mathrm{Ag}$ ) by an antigenspecific T-cell is followed by processing ( 1 ) and presentation in the context of MHC class II molecules. Recognition of this complex by the T-cell receptor (TCR) of the responding Th2 cell induces (2) CD40L expression. CD40L-induced aggregation of CD40 then triggers expression (3) of B7 (CD80). Subsequent interaction of B7 with CD28 on the surface of the T cell delivers a costimulatory signal (4) that amplifies cytokine synthesis (5) and the $\mathrm{T}$ cell-proliferative response. IL-4 produced in response to the synergistic TCR and CD28 signals binds to IL-4R (signal 1), which, in conjunction with CD40 ligation (signal 2), triggers the IgE isotype switch, B-cell proliferation, and expansion of the IgE-producing clone.

$\mathrm{\kappa B}) / \mathrm{p} 50$ and for one or more members of the C/EBP family of transcription factors. Mice deficient for NF$\mathrm{\kappa B} / \mathrm{p} 50$ are severely deficient in switching to IgE. The B cell-specific activator protein BSAP ( $\mathrm{Pax} 5$ ) also binds to the I $\varepsilon$ promoter, and has been implicated in activating $\mathrm{C} \varepsilon$-germline transcription.

The structure and function of $\varepsilon$-germline $m R N A$. The binding of IL-4 and IL-13 to their receptors on B cells delivers the first signal for isotype switching - the induction of germline transcripts at the $\mathrm{C} \varepsilon$ locus. These transcripts originate from a $5^{\prime}$ promoter of the $\mathrm{I} \varepsilon$ exon, which is located just upstream of the $4 \mathrm{C} \varepsilon$ exons. After processing, the mature germline mRNA's include the 140-bp I $\varepsilon$ exon and exons CE1-Ce4. These transcripts have been referred to as "sterile," because of the presence of stop codons in each of the 3 reading frames of IE.

Studies using mice with mutations affecting germline transcription have revealed that this process is absolutely required before isotype switching. Targeted deletion of the I $\varepsilon$ exon and its promoter at the IgG1 and IgG2b loci completely blocks switching to the these isotypes in mutant B cells. Conversely, insertion of a constitutively active $\mathrm{V}_{\mathrm{H}}$ promoter-IgH enhancer cassette in place of the I $\varepsilon$ promoter results in a modest enhancement of $\operatorname{IgE}$ switch recombination, and replacement of the IL4-responsive I $\gamma 1$ promoter with an LPS-inducible metallothionein $\mathrm{II}_{\mathrm{A}}$ promoter is sufficient to direct LPS-driven $\operatorname{IgG} 1$ switching $(15,16)$. Additional investigations suggest that the process of transcription, rather than the resultant mRNA transcript, facilitates the deletional switch recombination event. Harriman et al. analyzed IgA switching using mice retaining a normal I $\alpha$ promoter in which the I $\alpha$ exon was replaced by an HPRT minigene, and found that switching can occur to the locus despite the absence of complete I $\alpha$-containing transcripts (17). Similarly, we have observed that switch recombination can occur to the $\mathrm{C} \varepsilon$ locus when the I $\varepsilon$ exon and promoter are left intact, despite the absence of $\mathrm{C} \varepsilon$ exons in the germline transcripts. Taken together, these observations are consistent with a model in which IL-4/IL-13-driven transcription originating at the I $\varepsilon$ promoter alters the $\varepsilon$ heavy chain locus in a way that permits isotype switch recombination, while the germline transcripts probably serve no specific function.
CD40/CD40L interaction: the second signal for IgE isotype switch recombination. CD40L, which is absent on resting $\mathrm{T}$ cells, appears on their surface after T-cell receptor (TCR) activation by antigen/MHC (Figure 2). Binding of the newly expressed CD40L with CD40 on B cells provides the second signal for the induction of deletional switch recombination to IgE. A number of observations have pointed to a critical role for CD40/CD40L interactions in isotype switching to IgE. IgE production by IL-4-stimulated B cells in culture requires the presence of $\mathrm{T}$ cells. Anti-CD40 antibodies, which directly activate CD40L, can completely substitute for this T-cell help (18). Soluble human CD40 inhibits CD40/CD40L interaction, and blocks IL-4-driven IgE synthesis in such cultures. Patients with the X-linked hyper-IgM syndrome (XHIM) are deficient in CD40L; as a result, their B cells are unable to undergo isotype switching to produce $\operatorname{IgG}$, IgA, and $\operatorname{IgE}(19)$. Similarly, mice with targeted disruption of the CD40L or CD40 genes lack serum IgE, and their B cells fail to undergo isotype switching in vivo and in vitro after immunization with $\mathrm{T}$ cell-dependent antigens.

The unique ability of CD40 to trigger isotype switching in B cells suggests that it utilizes a distinct set of signaling pathways. Four intracellular proteins belonging to the family of TNF receptor-associated factors (TRAFs) TRAF-2, TRAF-3, TRAF-5, and TRAF- 6 - are known to associate with the intracytoplasmic domain of CD40 after its multimerization by interaction with CD40L. TRAF-2, TRAF-5, and TRAF- 6 can drive the activation of NF- $\kappa B$, which can then synergize with IL-4-derived signals, such as STAT6, on the Ce promoter. Although the function of TRAF-3 is not known, TRAF-3-deficient mice survive poorly and have a defect in isotype switching. In addition to triggering TRAF associations, engagement of CD40 activates protein tyrosine kinases (PTKs), which play an important role in immunoglobulin class switching. Box 1 and box 2 motifs, conserved sequences present in the intracellular domains of receptors that signal via JAK kinases, are present in CD40. JAK3 is constitutively associated with CD40, and CD40 engagement induces the tyrosine phosphorylation and activation of JAK3 and STAT3 (20).

Given its central role in the induction of the isotype switch, CD40 provides an attractive target for the phar- 
macological inhibition of $\operatorname{IgE}$ production in allergic patients. The widely used anti-asthma drugs disodium cromoglycate and nedocromil sodium inhibit IgE production by peripheral blood mononuclear cells as well as by highly purified B cells treated with anti-CD40 and IL4 (21). This inhibition occurs at the level of the B cell. Although neither compound affects IL-4-driven Cegermline transcription, both agents inhibit deletional switch recombination.

Deletional switch recombination. After the delivery of both IL-4 and CD40 signals, deletional switch recombination occurs (22). In IgE isotype switching, DNA breaks are introduced into the switch $(\mathrm{S})$ regions upstream of the $\mathrm{C} \mu$ gene $(\mathrm{S} \mu)$ and upstream of $\mathrm{C} \varepsilon(\mathrm{S} \varepsilon)$. The 2 switch regions are then ligated to form an $\mathrm{S} \mu / \mathrm{S} \varepsilon$ junction with deletion of the intervening DNA. This rearrangement brings the $\mathrm{V}_{\mathrm{H}}(\mathrm{D}) \mathrm{J}_{\mathrm{H}}$ segments encoding the antigen binding site into the immediate proximity of the $\mathrm{C} \varepsilon$ exons encoding the constant domains. The product of this recombination is the de novo generation of a complete multi-exon gene that can be transcribed as a single message encoding the full $\varepsilon$-heavy chain.

The molecular events in IgE isotype switching are not fully understood. It has been proposed that IL-4-driven germline transcription at the I $\varepsilon$ promoter makes the $\mathrm{S} \varepsilon$ sequences accessible to the switch recombinase, a putative complex of enzymes that mediates the DNA cutting and ligation reactions. Recombination enzymes appear to be constitutively active in B cells. Artificial substrates containing DNA sequences that encompass murine $S \mu$ and $S \gamma 2$ a undergo deletional switch recombination when integrated into the genome of murine B-cell lines. The switch recombinase may include the DNA repair enzyme DNA-dependent protein kinase (DNA-PK), which is defective in severe combined immunodeficiency (SCID) mice, and its associated DNA-binding proteins Ku70 and Ku80. The potential role of the DNA-PK complex in isotype switching is indicated by failure of B-cell precursors from SCID mice to undergo $S \mu / S \varepsilon$ recombination after stimulation with anti-CD40 (23), despite their normal expression of $\varepsilon$-germline transcripts. In addition, levels of $\mathrm{Ku}$ in B-cell nuclear extracts are greatly enhanced after CD40 ligation and IL-4 treatment.

$\mathrm{CD}^{+}$Th2 cells provide the signals for IgE production. IL-4 and CD40L, the 2 signals required for IgE production, are provided by $\mathrm{CD} 4^{+} \mathrm{Th} 2$ cells present in respiratory mucosa and regional lymphoid tissues of individuals with asthma. Th activation and expansion are accompanied by differentiation from a pluripotent Th0 state toward the Th1 or Th2 phenotypes, which are distinguished by their patterns of cytokine production. Th2 cells produce IL-4, IL-5, IL-6, IL-9, IL-10, IL-13, and GMCSF. Animal models provide evidence that Th2 cells are critical to the maintenance of the allergic bronchial inflammation typical of asthma and for allergen-evoked BHR. Allergen-specific Th2 cells can mediate the passive transfer of allergic airway responses. Th2-derived IL-4 and IL-13 drive germline transcription at the C $\varepsilon$ exons, thereby promoting isotype switching to IgE. After antigen encounter and TCR activation, they also express $\mathrm{CD} 40 \mathrm{~L}$, and thus are able to provide B cells with both of the signals necessary for IgE production. In addition, Th2 cells, via IL-5 and GM-CSF, promote eosinophilopoiesis, thereby enhancing allergic inflammation.

At the molecular genetic level, the commitment of undifferentiated $\mathrm{CD} 4^{+}$lineage cells to the Th1 or Th 2 phenotype involves an initial phase of chromatin remodeling, resulting in enhanced accessibility of cytokine gene loci. This is followed by the transcriptional activation of the Th1- or Th2-specific sets of cytokine genes under the control of transcription factors such as nuclear factor of activated T cells (NF-AT) and STATs. At the same time, a second wave of transcription factors is induced, including GATA-3 and c-Maf in the case of Th2 cells. It has been proposed that these might bind to regulatory sequences, recruit chromatin remodeling enzymes, and lead to stably inherited changes - as also occurs in other developmental pathways, including erythroid development. Finally, the stability of Th1 or Th2 expansion is maintained both by the autocrine effects of the cytokines IL-4 (Th2) and IFN- $\gamma$ (Th1) and the cross-inhibitory effects of these molecules on the development of the opposing lineage. The molecular genetic events involved in Th2 differentiation and stabilization are reviewed in detail by Ray and Cohn in this Perspectives series.

Antigen presentation in the asthmatic lung: initiation of Th2 responses in the atopic bronchial mucosa. Superimposed upon the above-discussed general influences controlling cytokine transcription and Th2 differentiation are specific features of the pulmonary cellular microenvironment in atopic individuals that may promote Th2 responses and IgE production in the bronchial mucosa (Figure 1). After allergen inhalation, the induction of an immune response requires uptake and processing by antigen-presenting cells (APCs), which then present peptide fragments to specific T cells. The type of APC, the expression of costimulatory molecules, and the secretion of cytokines can all sway the Th phenotype of the resultant T-cell response. In addition, neighboring cells, including mast cells, NK1.1 T cells, and NK1.1 non-T cells, might polarize the local cytokine milieu in a way that favors Th2 responses. Preexisting allergen-specific IgE may favor the induction of Th2 responses, both by activating mast cells to produce IL-4 and IL-13 and by facilitating B-cell antigen presentation.

Perhaps the most potent APCs in the lung are dendritic cells. After ingestion of antigen, these cells residing in the bronchial mucosa migrate to regional lymphoid tissues. This anatomic translocation is accompanied by increased surface expression of MHC class II antigens and B7-1/B7-2, along with the acquisition of potent antigen-presenting capabilities. The differential expression of some other markers, however, has defined dendritic cell subsets. CD8-CD11 bright myeloid dendritic cells are potent Th2 inducers. Another dendritic cell type, derived by culture of plasmacytoid $\mathrm{CD} 4{ }^{+} \mathrm{CD} 3-\mathrm{CD} 11 \mathrm{c}^{-}$cells with IL-3, is also an efficient Th2 inducer (24).

There is evidence to suggest that dendritic cell quantity and phenotype are altered in atopic individuals in a way that promotes Th2 responses. Dendritic cell numbers are increased in the bronchoalveolar lavage fluid of atopic patients. They express higher levels of HLA-DR, and have an enhanced capacity to present allergen to T cells and elic- 
it the production of IL-4 and IL-5. Cultured dendritic cells, prepared from atopic patients by incubation of monocytes with IL-4 and GM-CSF, have also been shown to have enhanced antigen-presenting capacity, and express HLADR, CD11b, and the high-affinity IgE receptor FcERI (25).

IgE production and Th2 responses following the interaction of T cells with APCs in the respiratory mucosa may be amplified by IL-4 and IL-13 produced by resident mast cells (26). These bone marrow-derived cells, which are widely distributed in mucosal and connective tissues, act as sentinels at sites of initial antigen exposure and are classically associated with the immediate-hypersensitivity reactions that are induced after antigen cross-linking of $\operatorname{IgE}$ bound to high-affinity receptors on their surface. Histamine, heparin, proteases, and arachidonic acid metabolites (prostaglandins and leukotrienes) are all important mast cell-derived effectors of airway edema, bronchoconstriction, and mucous secretion. A more important but perhaps underappreciated role of these mucosal sentinel cells, however, may be the maintenance of the Th2 shift in states of repetitive low-dose allergen exposure. In the presence of preformed, specific IgE, allergen-exposed mast cells are stimulated to transcribe Th2 cytokines, including IL-4, providing a powerful local amplification of the allergic response. In some murine asthma models, Kit ${ }^{\mathbb{W}} / K^{\mathrm{W}} \mathrm{t}^{\mathrm{W}-\mathrm{v}}$ mast cell-deficient mice have a significant reduction in eosinophil recruitment to the airway.

Another potential early source of IL-4 in evolving allergic responses is a minor subset of $\mathrm{CD}^{+} \mathrm{T}$ cells distinguished by their expression of the NK marker NK1.1. These $T$ cells are unusual in their expression of a strongly biased TCR $\alpha \beta$ repertoire and restriction by the nonclassical MHC class I molecule CD1. Cells with this phenotype are known to be capable of producing large amounts of IL-4 in vivo immediately after stimulation with anti-CD3 antibodies. However, their contribution to allergic responses remains unclear. Mice overexpressing NK1.1 cells have enhanced IL-4 production and increased levels of IgE and IgG1, both markers of Th2 responses; animals treated with anti-NK1.1 antibodies have suppressed Th2 responses. However, animals with a targeted mutation of $\beta_{2}$-microglobulin (an obligate component of CD1) are deficient in NK1.1 ${ }^{+} \mathrm{T}$ cells but appear to express functional Th2 responses to allergic and parasitic challenges. An NK1.1+ non-T cell population in mucosal tissues may also be important in driving Th2 responses, because CD11d-deficient mice that lack NK1.1 T cells also develop normal allergic responses, but such reactions are suppressed by pretreatment with anti-NK1.1 antibodies.

Genetic and environmental factors in the predisposition to atopy. About $5 \%$ of the population has a tendency toward a misguided, harmful immune response in the bronchial mucosa following allergen inhalation. Clearly, asthma runs in families in a complex pattern of inheritance consistent with polygenic influences. Genome-wide screens, linkage analysis, and studies of polymorphisms in candidate genes have all identified genetic factors likely to contribute to allergic pathogenesis. Nonspecific tendencies to produce high total levels of IgE are linked to 5q31-33, a locus that encodes multiple Th2 cytokines (27). Clinical atopy and the production of allergen-specific IgE have been linked to 11q12-13 and may be relat- ed to polymorphisms of the FceRI $\beta$ chain encoded there (28). As with other antigens, responses to allergens are modulated by the inheritance of particular MHC class II alleles or TCR variable-region repertoires. Nonimmunological host genetic factors important in the clinical syndrome of asthma have been identified as well. $\beta$-adrenergic receptor polymorphisms are associated with BHR and may be linked to nocturnal asthma. Bronchial responsiveness is probably affected by a number of genes. Murine studies have documented the presence of at least 3 independent loci controlling this trait (29).

Environmental factors are critical in asthma. Monozygotic twins have been shown to have asthma concordance rates of less than $20 \%$. Allergen exposure is a sine qua non for the development of allergic inflammation. The cumulative inhaled dose of sensitizers such as dust mite allergen over many years is directly related to the chance of developing allergen-specific IgE and asthma. Therefore, exposure alone is likely a major factor in asthma pathogenesis (30). Not all inhaled substances elicit an allergic response, and the major allergens have structural and functional properties that promote Th2 responses. Some allergenic proteins are toxic to respiratory epithelium and can actually disrupt tight junctions. Others have been shown to directly modulate host immune function by specific modification of immunocyte receptors, including proteolysis of CD23 (the low-affinity $\operatorname{IgE}$ receptor) and $\mathrm{CD} 25$ (the $\alpha$ chain of the IL-2 receptor).

Inflammatory or immunological processes arising in the bronchial mucosa coincident with or preceding allergen encounter probably also sway developing allergic reactions. Some viruses, including respiratory syncytial virus, may promote a shift toward Th2 expansion. Conversely, a propensity for Th1 responses in bronchial lymphoid tissue may be established after infection with other pathogens. Epidemiologic data from regions of the world in which mycobacterial infection is endemic indicate that mycobacterial infection, which results in Th1 activation, protects patients from development of bronchial allergy. Such associations have given rise to the "hygiene" hypothesis, in which some investigators have speculated that increasing antibiotic use and resultant alterations in the microbiology of the respiratory mucosa result in decreased local production of the Th1 cytokines IL-12 and IFN- $\gamma$ and may be responsible for the increase in asthma rates in developed nations.

Conclusions. The tendency to produce IgE and express the clinical features of the atopic triad results both from an inborn predisposition toward allergic responses and from chronic exposure to environmental allergens. It is clear that susceptibility to allergy is associated with the enhanced production of the allergic cytokines IL-4, IL-5, and IL-13, leading both to the production of $\operatorname{IgE}$ and induction of eosinophil-predominant inflammation. Driving the allergic response are $\mathrm{CD}^{+}{ }^{+} \mathrm{Th} 2 \mathrm{~T}$ cells, whose activation and differentiation arise out of the interaction of a large number of cellular molecular factors (reviewed above). One can anticipate that the genetic and environmental factors that predispose some of us toward the Th2 responses that underlie the atopic diseases will be better understood, and that the next few years will yield new approaches to regulating this central cause of allergy. 


\section{Acknowledgments}

This work is supported by National Institutes of Health grants AI-42202, AI-31136, and AI-31541, and by the Baxter, Olsten, and Centeon corporations. H.C. Oettgen is funded by a Pew Scholarship in the Biomedical Sciences, and by the Asthma and Allergy Foundation of America.

1. Postma, D.S., et al. 1995. Genetic susceptibility to asthma - bronchial hyperresponsiveness coinherited with a major gene for atopy. N. Engl. J. Med. 333:894-900.

2. Heyman, B., Tianmin, L., and Gustavsson, S. 1993. In vivo enhancement of the specific antibody response via the low-affinity receptor for IgE. Eur. J. Immunol. 23:1739-1742.

3. Yamaguchi, M., et al. 1997. IgE enhances mouse mast cell FceRI expression in vitro and in vivo. Evidence for a novel amplification mechanism for IgE-dependent reactions. J. Exp. Med. 185:663-672.

4. Kisselgof, A.B., and Oettgen, H.C. 1998. The expression of murine B cell $\mathrm{CD} 23$, in vivo, is regulated by its ligand, IgE. Int. Immunol. 10:1377-1384.

5. Mehlhop, P.D., et al. 1997. Allergen-induced bronchial hyperreactivity and eosinophilic inflammation occur in the absence of IgE in a mouse model of asthma. Proc. Natl. Acad. Sci. USA. 94:1344-1349.

6. Oettgen, H.C., et al. 1994. Active anaphylaxis in IgE-deficient mice. Nature. 370:367-370.

7. Miyajima, I., et al. 1997. Systemic anaphylaxis in the mouse can be mediated largely through IgG1 and Fc gammaRIII. Assessment of the cardiopulmonary changes, mast cell degranulation, and death associated with active or IgE- or IgG1-dependent passive anaphylaxis. J. Clin. Invest. 99:901-914.

8. Boulet, L.P., et al. 1997. Inhibitory effects of an anti-IgE antibody E25 on allergen-induced early asthmatic response. Am. J. Respir. Crit. Care Med. 155: $1835-1840$

9. Fahy, J.V., et al. 1997. The effect of an anti-IgE monoclonal antibody on the early- and late-phase responses to allergen inhalation in asthmatic subjects. Am. J. Respir. Crit. Care Med. 155:1828-1834.

10. McKenzie, G.J., Fallon, P.G., Emson, C.L., Grencis, R.K., and McKenzie, A.N. 1999. Simultaneous disruption of interleukin (IL)-4 and IL-13 defines individual roles in $\mathrm{T}$ helper cell type 2-mediated responses. J. Exp. Med. 189:1565-1572.

11. Wills-Karp, M., et al. 1998. Interleukin-13: central mediator of allergic asthma. Science. 282:2258-2261.

12. Grunig, G., et al. 1998. Requirement for IL-13 independently of IL-4 in experimental asthma. Science. 282:2261-2263.

13. Shimoda, K., et al. 1996. Lack of IL-4-induced Th2 response and IgE class switching in mice with disrupted Stat6 gene. Nature. 380:630-633.

14. Hershey, G., Friedrich, M., Esswein, L., Thomas, M., and Chatila, T. 1997.
The association of atopy with a gain-of-function mutation in the $\alpha$ subunit of the interleukin-4 receptor. N. Engl. J. Med. 337:1720-1725.

15. Bottaro, A., et al. 1994. S region transcription per se promotes basal IgE class switch recombination but additional factors regulate the efficiency of the process. EMBOJ. 13:665-674.

16. Lorenz, M., Jung, S., and Radbruch, A. 1995. Switch transcripts in immunoglobulin class switching. Science. 267:1825-1828.

17. Harriman, G.R., Bradley, A., Das, S., Rogers-Fani, P., and Davis, A.C. 1996. IgA class switch in I alpha exon-deficient mice. Role of germline transcription in class switch recombination. J. Clin. Invest. 97:477-485.

18. Jabara, H., Fu, S., Geha, R.S., and Vercelli, D. 1990. CD40 and IgE: synergism between anti-CD40 monoclonal antibody and interleukin 4 in the induction of IgE synthesis by highly purified human B cells. J. Exp. Med. 172:1861-1864.

19. Bacharier, L.B., Jabara, H., and Geha, R.S. 1998. Molecular mechanisms of immunoglobulin E regulation. Int. Arch. Allergy Immunol. 115:257-269.

20. Hanissian, S.H., and Geha, R.S. 1997. Jak3 is associated with CD40 and is critical for CD40 induction of gene expression in B cells. Immunity. 6:379-388.

21. Loh, R., Jabara, H., and Geha, R.S. 1994. Disodium cromoglycate inhibits $\mathrm{S} \mathrm{mu} \rightarrow \mathrm{S}$ epsilon deletional switch recombination and IgE synthesis in human B cells. J. Exp. Med. 180:663-671

22. Shapira, S., Vercelli, D., Jabara, H., Fu, S., and Geha, R.S. 1992. Molecular analysis of the induction of Immunoglobulin E synthesis by human B cells by interleukin 4 and engagement of CD40 antigen. J. Exp. Med. 175:289-292.

23. Rolink, A., Melchers, F., and Andersson, J. 1996. The SCID but not the $R A G-2$ gene product is required for $\mathrm{Sv}-\mathrm{S} \varepsilon$ heavy chain class switching. Immunity. 5:319-330.

24. Rissoan, M.C., et al. 1999. Reciprocal control of T helper cell and dendritic cell differentiation. Science. 283:1183-1186.

25. van den Heuvel, M.M., Vanhee, D.D., Postmus, P.E., Hoefsmit, E.C., and Beelen, R.H. 1998. Functional and phenotypic differences of monocytederived dendritic cells from allergic and nonallergic patients. J. Allergy Clin. Immunol. 101:90-95.

26. Galli, S.J. 1997. Complexity and redundancy in the pathogenesis of asthma: reassessing the roles of mast cells and T cells. J. Exp. Med. 186:343-347.

27. Marsh, D.G., et al. 1994. Linkage analysis of IL4 and other chromosome 5 q31.1 markers and total serum immunoglobulin E concentrations. Science. 264:1152-1156.

28. Cookson, W.O., et al. 1992. Maternal inheritance of atopic IgE responsiveness on chromosome 11q. Lancet. 340:381-384.

29. De Sanctis, G.T., et al. 1995. Quantitative locus analysis of airway hyperresponsiveness in A/J and C57BL/6J mice. Nat. Genet. 11:150-154.

30. Platts-Mills, T.A., and Carter, M.C. 1997. Asthma and indoor exposure to allergens. N. Engl. J. Med. 336:1382-1384. 